\title{
Article
}

\section{Ethical Considerations in Multiple Pregnancy: Preterm Delivery in the Setting of Discordant Fetal Anomaly}

\author{
Thérèse Phillips ${ }^{1}$, Bryanna Moore ${ }^{2}$, Elske Posma ${ }^{3}$, Lynn Gillam ${ }^{2,4}$, Rocco Cuzzilla ${ }^{3,5}$ and Stephen Cole ${ }^{1}$ \\ ${ }^{1}$ Multiple Pregnancy Clinic, The Royal Women's Hospital, Melbourne, Victoria, Australia, ${ }^{2}$ Children's Bioethics Centre, The Royal Children's Hospital, Melbourne, \\ Victoria, Australia, ${ }^{3}$ Fetal Medicine Unit, The Royal Women's Hospital, Melbourne, Victoria, Australia, ${ }^{4}$ Melbourne School of Population and Global Health, \\ The University of Melbourne, Melbourne, Victoria, Australia and ${ }^{5}$ Neonatal Services, The Royal Women's Hospital, Melbourne, Victoria, Australia
}

\begin{abstract}
Planning for the preterm birth of a fetus with known anomalies can raise complex ethical issues. This is particularly true of multiple pregnancies, where the interests of each fetus and of the expectant parent(s) can conflict. In these complex situations, parental wishes and values can also conflict with the recommendations of treating clinicians. In this article, we consider the case of a dichorionic twin pregnancy complicated by the diagnosis of vein of Galen aneurysmal malformation (VGAM) in one of the twins at 28 weeks' gestation. Subsequent deterioration of the affected twin prompted the parents to request preterm delivery to prevent the imminent in-utero demise of the affected twin. However, given the associated risks of prematurity, complying with the parents' request may have disadvantaged the health and wellbeing of the unaffected twin. This article canvases the complex ethical issues raised when parents request preterm delivery of a multiple pregnancy complicated by a fetal anomaly in one twin, and the various ethical tools and frameworks that clinicians can draw on to guide their decision-making in such cases.
\end{abstract}

Keywords: Perinatal loss; bereavement; complex grief; twin pregnancy; DCDA; multiple pregnancy; perinatal ethics; obstetric ethics

(Received 6 April 2018; accepted 12 March 2019)

A 38-year-old gravida 5 para 2 with a spontaneously conceived dichorionic diamniotic (DCDA) twin pregnancy was referred to our tertiary centre for complex antenatal care following the finding of a vein of Galen aneurysmal malformation (VGAM) in one fetus at 28 weeks' gestation. VGAM is a rare congenital anomaly of the cerebral vasculature with a poor prognosis, particularly in the presence of associated brain anomalies (Deloison et al., 2012; Paladini et al., 2017). Perinatal death from high-output cardiac failure is almost certain if the VGAM remains untreated (Jones et al., 2002; Khullar et al., 2010). The expectant parents were counseled regarding risks associated with VGAM in the affected twin and the clinicians' expectation of delivery at term. The parents were also counseled about the potential for selective reduction of the affected twin, which they declined.

On further imaging, the affected twin showed progressively worsening neurologic and cardiac sequelae of the VGAM, including marked cerebral ventriculomegaly and cardiac failure. By 34 weeks' gestation, the affected twin showed evidence of reversed end-diastolic flow in the umbilical artery, low resistance in the middle cerebral artery and an abnormal wave pattern in the ductus venosus, suggesting that death was likely to be imminent. Following discussion, the parents requested preterm delivery to prevent in-utero demise of the affected twin. The parents' rationale was to afford them the opportunity to meet their live-born twins

Author for correspondence: Dr Stephen Cole, Email: Steve.Cole@thewomens.org.au Cite this article: Phillips T, Moore B, Posma E, Gillam L, Cuzzilla R, and Cole S. (2019) Ethical Considerations in Multiple Pregnancy: Preterm Delivery in the Setting of Discordant Fetal Anomaly. Twin Research and Human Genetics 22: 120-123, https:// doi.org/10.1017/thg.2019.12 and their perception that intrauterine fetal death (FDIU) of the affected twin could cause psychological harm to the unaffected co-twin. They also felt that it would impact negatively on their and other family members' interactions with the surviving twin. The parents' request for preterm delivery raised complex ethical considerations with respect to the potential harm to the unaffected twin and the competing interests of multiple fetuses and the parents.

\section{Discussion}

The parents' request for delivery at 34 weeks' gestation in this case raised concerns regarding the inherent risks of prematurity for the unaffected twin. Moderate preterm birth $(32$ to $<34$ weeks' gestation) and late preterm delivery ( 34 to $<37$ weeks) is associated with a relatively low risk of mortality compared with infants who are born at less than 32 weeks' gestation (Cheong \& Doyle, 2012). However, when compared with term-born children, they have both an increased mortality and in-hospital morbidity (Cheong \& Doyle, 2012). While the mortality of infants born prematurely decreases exponentially closer to term, moderate and late preterm infants concerningly still have a mortality rate five times that of term-born infants (Li et al., 2011). However, late preterm birth often occurs in the setting of pregnancy complications over concerns about fetal or maternal wellbeing that were not pertinent to this case. Considering morbidity, late preterm infants have a greater likelihood of admission to a neonatal unit to manage the expected and potential problems of prematurity, including respiratory distress syndrome, hypothermia, hypoglycemia and the 
Table 1. A model for ethical decision-making

\begin{tabular}{ll}
\hline Step & \multicolumn{1}{c}{ Process } \\
\hline 1 & Clearly state the problem and consider its context. \\
\hline 2 & Get the facts. \\
\hline 3 & Consider (core) ethical principles. \\
\hline 4 & Consider the problem from another perspective or theory. \\
\hline 6 & Identify ethical conflicts. \\
\hline 7 & Consider the law. \\
\hline
\end{tabular}

Source: Adapted from p. 94 Kerridge et al. (2009).

establishment of suck feeding (Cheong \& Doyle, 2012). There is also increasing evidence that moderately preterm infants are at risk of adverse neurodevelopmental outcomes into adolescence and adulthood, including poorer school performance, cognitive and language impairments, and psychiatric conditions (Cheong \& Doyle, 2012; Cheong et al., 2017).

While parents commonly advocate for the best interests of their children, parental requests regarding unborn children can be complex, especially if a parental request has the potential to lead to a suboptimal, or even harmful, outcome for the child. Given that the risks of prematurity at 34 weeks' gestation were, in this case, considered relatively low, the parents' wishes were given ethical weight and the preterm delivery of their infants was supported, for the reasons described below. In this case, the treating clinicians drew on a number of ethical tools and frameworks to guide their decision-making (see Table 1).

An ethical decision-making model from Kerridge et al. (2009) was used as an initial guide. The treating clinicians evaluated relevant clinical information and listened to the parents' request and expressed value system. The team assessed the parents' rationale for their request and their understanding of the relevant issues. The clinicians then considered a common approach to addressing ethical dilemmas in clinical decision-making, the application of Beauchamp and Childress's (2013) four principles: autonomy, beneficence, nonmaleficence and justice (Beauchamp \& Childress, 1979, 2013). Justice requires fair distribution of resources. The foreseeable need for a special care admission and neonatology resources was considered, acknowledging that this could have been prevented had the family accepted a term delivery. Nonmaleficence requires clinicians not to cause harm. Beneficence refers to clinicians 'doing good' and overlaps with the duty of nonmaleficence. Beneficence for the parents in this case primarily involved assuaging their anxieties and addressing their perception that the surviving twin may be negatively impacted by the fetal death in-utero. In relation to beneficence and nonmaleficence for the unaffected twin, the key issue is the effect of any decision on the future child (Wilkinson et al., 2016). It was agreed that the risk of morbidity and long-term adverse neurodevelopment would be mitigated by delivery closer to term. However, respect for parental autonomy was a competing consideration. The team recognized that respect for parental autonomy meant not merely accepting parents' wishes but engaging them in rational moral dialogue (Savulescu, 1995, 1997). While the parents' views may not have mirrored the treating team's, it is ethically problematic to assume that the parents are therefore being irrational. Therefore, attempts at engaging the parents in open discussion about their reasons and the clinicians' reasons were deemed ethically appropriate.
The clinicians' reasons for recommending continuing the pregnancy to full term after the expected death in-utero of the affected twin were explained and discussed with parents. The key issues were the risks to the unaffected twin of premature delivery (as detailed above), and the lack of evidence for any negative psychological risk to the unaffected twin from the death in-utero of the other twin. There are minimal risks to the surviving twin following co-twin demise in DCDA twins (Hillman, Morris, \& Kilby, 2011). Consequently the parents were reassured that single death in utero of twin 1 could not affect the health of the surviving twin.

The approach of taking the parents' views seriously is also supported by the concept of Patient and Family-Centred Care (PFCC; Zlotnik Shaul, 2014). The PFCC approach to health care is based on four key concepts or processes, including information sharing, dignity and respect, participation and collaboration (Johnson et al., 2009; Zlotnik Shaul, 2014). Understanding the thought processes that inform parental decision-making can be helpful when considering to what extent this process constitutes an autonomous decision based on their value system, and consequently how much emphasis should be placed on balancing parental requests against other ethical principles.

Evans et al. (2014) argue that parents interpret and process information to balance decision-making within a sociological value-based construct or style-based method referred to as 'framing.' Within this construct, they detail three styles of framing: medical, conceptional and lifestyle. Medical framing concerns facts, such as results of investigations, prognosis, preterm delivery outcomes; conceptional framing concerns normative and moral values and beliefs concerning the beginning of life and expectations following birth; and lifestyle framing focuses on the family as a whole (Evans \& Britt, 2010). According to Fletcher (2004), most parents will consider a combination of all three frames during their decision-making in order to '[try] to get the most benefit for the least harm' (Evans et al., 2004; Evans \& Britt, 2010, p. 299). In keeping with this view and considered against Evan and Britt's stylebased method, the parents' request for preterm delivery in this case and the concerns they expressed regarding the potential impact that this may have on their family, both then and in the future, illustrated the application of all three frames: the medical (weighted prematurity risks and primarily the psychological wellbeing of Twin 2), the conceptional (two twins being born alive together) and lifestyle frames (family as a unit). The parents advocated for the 'unit identity' of their unborn twins, respecting each child's 'atomistic' and 'relational' or social autonomy (Tauber, 2003 , p. 489), and gave due consideration to the best interests of their family unit. This was separate to their request for the opportunity to provide family-centered palliative care and to have a liveborn delivery of both twins (medical and conceptional framing). Ultimately, the underlying differences in opinion between parents and clinicians regarding early versus full-term delivery were not resolved. Following discussion within the treating team, it was agreed that the principles of nonmaleficence (to the unaffected twin) and justice did not significantly outweigh the principles of beneficence (to the parents) and autonomy as they applied in this case, and therefore that the parental request could be supported.

In making this decision, the treating team referred to the zone of parental discretion (ZPD) tool (Gillam, 2016). In the context of disagreement with parents, the ZPD tool for clinicians focuses on: (1) possible (significant) harm to child and (2) parents' moral claim to be decision-makers for the child. The harm principle has largely replaced the best-interests standard as the guiding standard in 
Table 2. The professional responsibility model of obstetric ethics

\begin{tabular}{|c|c|c|c|}
\hline \multicolumn{4}{|c|}{ Three models of obstetrics ethics } \\
\hline Variable & Fetal rights reductionism model & Professional responsibility model & $\begin{array}{l}\text { Pregnant women's rights } \\
\text { reductionism model }\end{array}$ \\
\hline Pregnant woman & $\begin{array}{l}\text { Pregnant woman's rights systematically } \\
\text { secondary to fetal rights }\end{array}$ & $\begin{array}{l}\text { Autonomy-based and beneficence } \\
\text { obligations }\end{array}$ & $\begin{array}{l}\text { Pregnant woman's rights systematically } \\
\text { override fetal rights }\end{array}$ \\
\hline Previable fetus & $\begin{array}{l}\text { Fetal rights systematically override } \\
\text { woman's rights }\end{array}$ & $\begin{array}{l}\text { Beneficence-based obligations if the } \\
\text { status of patienthood is determined } \\
\text { by the pregnant woman }\end{array}$ & $\begin{array}{l}\text { Fetal rights systematically secondary } \\
\text { to woman's rights }\end{array}$ \\
\hline Viable fetus & $\begin{array}{l}\text { Fetal rights systematically override } \\
\text { woman's rights }\end{array}$ & Beneficence-based obligations & $\begin{array}{l}\text { Fetal rights systematically secondary } \\
\text { to woman's rights }\end{array}$ \\
\hline
\end{tabular}

Source: Reprinted from Am J Obstet Gynecol 2011;205 Chervenak F, McCullough LB, Brent RL. The professional responsibility model of obstetrical ethics: avoiding the perils of clashing rights, p. 315.2 Copyright Mosby Inc. (2011), republished with permission from Elsevier.

pediatric medical decision-making. Parents have ethical and legal discretion to make decisions for their child that are not optimal for the child, provided that they are 'good enough,' and do not cause significant harm to the child (Diekema, 2004; Gillam, 2016). In this and other cases involving parental requests, there is often a range of ethically acceptable outcomes, as opposed to a single, ethically preferable outcome or best course of action. Essentially, according to the ZPD, parents' wishes should be respected unless there is a good reason not to (Gillam, 2016).

In this case, preterm delivery at 34 weeks was not deemed significantly harmful or life-threatening for Twin 2, who was still very likely to do well, and go on to have a life worth living. The risks of poor outcomes were small. The parents were engaged in discussions about both the short- and long-term morbidities associated with delivery at 34 weeks. In accordance with the ZPD, the treating team considered the impact on the parents and their surviving child of going against their wishes and refusing an early delivery request. The long-term risks and effects on psychological wellbeing following selective FDIU in multiple pregnancies, particularly for the surviving infant, are largely unknown (Evans et al., 2004; Evans \& Britt, 2010). However, there is clear evidence that the grief related to multiple pregnancy loss is complex for the surviving children and their families, although it is not known whether the nature or magnitude of that grief is different for antenatal (fetal) compared to postnatal (neonatal) loss (Bryan, 1999; Withrow \& Schwiebert, 2005). While there is paucity of literature regarding this, the treating team took this possibility seriously and factored it into their decision to respect the parents' wishes/request. After weighing up all these considerations, the team decided that the impact of overriding the parents' wishes in this case would not have been justified by the perceived very low risk of significant harm to the unaffected twin, and thus collectively decided against doing so.

Obstetrics is unique in that it is the only field in medicine where decisions made in the care of one person immediately affect the outcome of another' (Deshpande \& Oxford, 2012, p. e144). This unique situation lends itself to framing ethically challenging situations in terms of a clash in rights between the pregnant woman and the fetus, as is suggested in Chervernak's model (Table 2; Chervenak, McCullough, \& Brent, 2011). While in law, in Australia and in other countries, the fetus does not have any legal rights, potential harm to a future child as a direct result of antenatal decision-making is a strong morally relevant consideration (Wilkinson et al., 2016). This is enough to ground the idea that the fetus is also a patient with moral rights (Chervenak et al., 2011). However, framing in terms of rights leads to the fraught matter of resolving clashes between rights, and it is not at all clear how to do this. In the end, this leads to the sort of balancing of the interests of the fetus and the pregnant women that are described above. The team did not find use of a rights-based model helpful.

\section{Conclusion}

One notable feature of this case was that the parents' reasons for requesting preterm delivery were not reasons that the treating team had previously considered. This, coupled with the clinical complexity associated with managing a twin pregnancy complicated by fetal anomaly, meant that the treating team did not feel that one single ethical principle, tool or framework could provide sufficient guidance when deciding how to best care for both the infants and the parents. All of the above principles, tools and framework were considered in discussions and provided valuable insight into the complex ethical issues at stake.

In this case, the parents' wishes were respected, and a cesarean section was performed at 34 weeks' gestation following a course of antenatal corticosteroids to promote fetal lung maturation. Both infants were live born. The affected twin died shortly after birth as he was comforted in his parents' arms, surrounded by his family and surviving twin. The unaffected twin was born in good condition, required minimal support, and had an uncomplicated postnatal course with a short special care admission. At a postpartum debriefing, the parents expressed their gratitude for a delivery and palliative care plan that had respected their wishes. This case highlights the significance that respect for parents' wishes may have in grief preparation, experience and acceptance. On reflection, the clinicians believed that this case had a positive outcome. If the parents' wishes were for delivery at an earlier gestation, the outcome may have been different as the risk to the health and wellbeing of the unaffected twin would have been higher. Respect for parental autonomy should not automatically trump the interests of the fetus(es).

In conclusion, this case of a multiple pregnancy complicated by discordant fetal anomaly presented complex ethical considerations for the treating clinicians. Through ongoing consultation with the parents, and by considering a combination of ethical frameworks and accepting the possibility of more than one ethically acceptable outcome, clinicians were able to develop what they felt was an ethically and clinically acceptable care plan that balanced respecting 
the parents' values and autonomy with the interests of the unaffected, surviving twin. While the long-term effects on the psychological wellbeing of surviving children of multiple pregnancies following selective fetal death in-utero are not well documented in literature, postnatal feedback from the parents suggests that respecting the parents' wishes in this case will continue to have a lasting positive impact on the family.

Financial support. The authors have not received grants from any funding agency, commercial or not-for-profit sectors.

\section{Conflicts of interest. None.}

\section{References}

Beauchamp, T. L., \& Childress, J. (1979). Principles of biomedical ethics (1st ed.). New York, NY: Oxford University Press.

Beauchamp, T. L., \& Childress, J. (2013). Principles of biomedical ethics (7th ed.). New York, NY: Oxford University Press.

Bryan, E. (1999). The death of a twin. In A. C. Sandbank (Ed.), Twin and triplet psychology (pp. 186-200). London: Routledge.

Cheong, J. L., \& Doyle, L. W. (2012). Increasing rates of prematurity and epidemiology of late preterm birth. Journal of Paediatrics and Child Health, 48, 784-788.

Cheong, J. L., Doyle, L. W., Burnett, A. C., Lee, K. J., Walsh, J. M., Potter, C. R., ... Spittle, A. J. (2017). Association between moderate and late preterm birth and neurodevelopment and social-emotional development at age 2 years. JAMA Pediatrics, 171, e164805.

Chervenak, F. A., McCullough, L. B., \& Brent, R. L. (2011). The professional responsibility model of obstetrical ethics: Avoiding the perils of clashing rights. American Journal of Obstetrics \& Gynecology, 205, e311-e315.

Deloison, B., Chalouhi, G. E., Sonigo, P., Zerah, M., Millischer, A. E., Dumez, Y., ... Salomon, L. J. (2012). Hidden mortality of prenatally diagnosed vein of Galen aneurysmal malformation: Retrospective study and review of the literature. Ultrasound Obstet Gynecol, 40, 652-658.

Deshpande, N. A., \& Oxford, C. M. (2012). Management of pregnant patients who refuse medically indicated cesarean delivery. Reviews in Obstetrics and Gynecology, 5, e144-e150.

Diekema, D. S. (2004). Parental refusals of medical treatment: The harm principle as threshold for state intervention. Theoretical Medicine and Bioethics, $25,243-264$.

Evans, M., Kaufman, M., Urban, A., Britt, D., \& Fletcher, J. (2004). Fetal reduction from twins to a singleton: A reasonable consideration? Obstetrics \& Gynecology, 104, 102-109.
Evans, M. I., Andriole, S., \& Britt, D. W. (2014). Fetal reduction: 25 years' experience. Fetal Diagnosis and Therapy, 35, 69-82.

Evans, M. I., \& Britt, D. W. (2010). Multifetal pregnancy reduction: Evolution of the ethical arguments. Seminars in Reproductive Medicine, $28,295-302$.

Gillam, L. (2016). The zone of parental discretion: An ethical tool for dealing with disagreement between parents and doctors about medical treatment for a child. Clinical Ethics, 11, 1-8.

Hillman, S. C., Morris, R. K., \& Kilby, M. D. (2011). Co-twin prognosis after single fetal death: a systematic review and meta-analysis. Obstetrical Gynecology, 118, 928-940. doi: 10.1097/AOG.0b013e31822f129d

Johnson, B. H., Abraham, M. R., \& Shelton, T. L. (2009). Patient- and familycentered care: Partnerships for quality and safety. North Carolina Medical Journal, 70, 125-130.

Jones, B. V., Ball, W. S., Tomsick, T. A., Millard, J., \& Crone, K. R. (2002). Vein of Galen aneurysmal malformation: Diagnosis and treatment of 13 children with extended clinical follow-up. American Journal of Neuroradiology, 23, 1717-1724.

Kerridge, I., Lowe, M., \& Stewart, C. (2009). Ethics and law for health professionals (3rd ed.). Sydney, Australia: The Federation Press p. 94.

Khullar, D., Andeejani, A., \& Bulsara, K. (2010). Evolution of treatment options for vein of Galen malformations. Journal of Neurosurgery: Pediatrics, $6,444-451$.

Li, Z., McNally, L., Hilder, L., \& Sullivan, E. (2011). Australia's mothers and babies 2009. Sydney, Australia: Australian Institute of Health and Welfare.

Paladini, D., Deloison, B., Rossi, A., Chalouhi, G. E., Gandolfo, C., Sonigo, P., ... Salomon, L. J. (2017). Vein of Galen aneurysmal malformation (VGAM) in the fetus: retrospective analysis of perinatal prognostic indicators in a two-center series of 49 cases. Ultrasound in Obstetrics \& Gynecology, 50, 192-199.

Savulescu, J. (1995). Rational non-interventional paternalism: Why doctors ought to make judgments of what is best for their patients. Journal of Medical Ethics, 21, 327-331.

Savulescu, J. (1997). Liberal rationalism and medical decision-making. Bioethics, 11, 115-129.

Tauber, A. I. (2003). Sick autonomy. Perspectives in Biology and Medicine, 46, 484-495.

Wilkinson, D., Skene, L., De crespigny, L., \& Savulescu, J. (2016). Protecting future children from in-utero harm. Bioethics, 30, 425-432.

Withrow, R., \& Schwiebert, V. L. (2005). Twin loss: Implications for counselors working with surviving twins. Journal of Counseling \& Development, 83, 21-28.

Zlotnik Shaul, R. (2014). Paediatric patient and family-centred care: Ethical and legal issues. New York, NY: SpringerLink. 